\title{
Enhancement of Electrolyte Properties for High Energy Density Supercapacitors by using Additive Materials
}

\author{
Cheong Kim ${ }^{1}$, Hiroki Habazaki ${ }^{2}$, and Soo Gil Park ${ }^{1, *}$ \\ ${ }^{1}$ Department of Engineering Chemistry, Chungbuk National University, Chungdae-ro 1, Seowon, 28644, Korea \\ ${ }^{2}$ Graduate School of Chemical Sciences and Engineering, Hokkaido University, Sapporo, Hokkaido 060-8628, Japan
}

\begin{abstract}
In this study, we studied the enhancement of the energy densities of electrochemical capacitors by improving the working voltage range of the electrolyte. To prevent the decomposition of the electrolyte, stable SEI layers were formed by reductive degradation of additive materials such as fluoro-ethylene carbonate (FEC) and vinyl ethylene carbonate (VEC) before degradation of the base electrolyte. As a result, the solution resistance $\left(\mathrm{R}_{\mathrm{s}}\right)$ of EC:DMC + SL $20 \%+\mathrm{VEC} 1 \%$ electrolytes observed $1.47 \Omega$ and the charge transfer resistance $\left(\mathrm{R}_{\mathrm{ct}}\right)$ was $2.64 \Omega$ at the open circuit voltage. Additionally, a cycle retention of $94 \%$ was observed for EC:DMC + SL $20 \%+$ VEC $1 \%$ after 500 cycles at $3.5 \mathrm{~V}$.
\end{abstract}

Keywords : Electrolyte, Electrochemical capacitors, Additive materials

Received : 10 February 2016, Revised : 25 April 2016, Accepted : 25 April 2016

\section{Introduction}

Recently, high power batteries and devices have been of interest due to increases in the electronic vehicles industry [1,2]. Of the candidates, electrochemical capacitors, such as electrical double layer capacitors (EDLCs) and super-capacitors, have received substantial attention as large-scale energy storage devices due to their higher power densities and long cycle life compared to lithium ion batteries [3,4]. However, electrochemical capacitors have lower energy densities than lithium ion batteries. Energy density can be calculated using the following equation:

$$
\begin{aligned}
& \text { E. D. (Energy density) } \\
& =\frac{1}{2} \mathrm{CV}^{2}(\mathrm{C} \text { : capacitance, } \mathrm{V} \text { : potential window })
\end{aligned}
$$

According to Eqn. 1, increasing the voltage is the most effective method of improving the energy density [5]. To increasing the potential window of electrochemical capacitors, commercial devices use

*E-mail address: sgpark@cbnu.ac.kr

DOI: http://dx.doi.org/10.5229/JECST.2016.7.3.214 organic electrolytes such as $\mathrm{TEABF}_{4}$ in acetonitrile (AN) or propylene carbonate (PC), enabling voltages up to $2.7-2.8 \mathrm{~V}$ to be reached [6]. However, AN and $\mathrm{PC}$ are expensive, noxious and have low conductivities, so alternative materials are required. In addition, research into electrolytes with increased potential windows has been more successful at increasing energy density than work focused on commercial electrochemical capacitors [7]. In this study, we studied the enhanced the energy densities of electrochemical capacitors by using a composite solvent and inserting additive materials through the formation of stable SEI layers. We selected a composite solvent using ethylene carbonate (EC) and dimethyl carbonate $(\mathrm{DMC})$ (volume ratio $=1: 1)$ for the base electrolyte due to its higher dielectric constant and lower viscosity when compared to commercial electrolytes. Sulfolane (SL), which has high oxidative stability and good cycling ability at high voltage, was added to the composite solvent in the required amounts [8]. Additionally, fluoro-ethylene carbonate (FEC) and vinyl ethylene carbonate (VEC) were added to the composite solvent to allow formation of stable SEI layers. In the past few years, there have 
been many reports on FEC and VEC as electrolyte additives for improving the properties of SEI films because of their lower LUMO levels $(-0.12 \mathrm{eV}$ and $0.5 \mathrm{eV}$, respectively) and higher HOMO levels ($7.79 \mathrm{eV}$ and $-7.75 \mathrm{eV}$, respectively) than EC (LUMO: $0.86 \mathrm{eV}$ and HOMO: $-8.19 \mathrm{eV}$ ) [9]. This allows them to stable SEI layers by reacting before other materials, so the reductive decomposition of FEC and VEC to form SEI films can occur at higher potentials than is possible for of EC [10]. To study the effect of additive on electrolyte reactions, electrochemical measurements, such as cycle retention and c-rate capability, were carried out at high voltage $(>3.5 \mathrm{~V})$.

\section{Experimental Section}

\subsection{Preparation of electrode}

Electrode and additive materials were obtained from Pureechem. Co,. The electrode was prepared by coating a slurry of $80 \mathrm{wt} \%$ active carbon (CEP-17), $10 \mathrm{wt} . \%$ conductive agent (super-p) and $10 \mathrm{wt} . \%$ binders, which consisted of carboxymethyl cellulose (CMC, 1 wt.\%, Sigma-Aldrich), styrene butadiene rubber (SBR, 2 wt.\%, Sigma-Aldrich) and polytetrafluoro-ethylene (PTFE, 2 wt.\%, Sigma-Aldrich) on Al foil using the doctor blade technique. After the coating, the electrode was dried at $120^{\circ} \mathrm{C}$ for $24 \mathrm{~h}$ in air.

\subsection{Preparation of electrolyte}

The base electrolyte was ethylene carbonate (EC) and dimethyl carbonate (DMC) [EC:DMC (vol. ratio $=1: 1)]$ with $1 \mathrm{M} \mathrm{SP}_{2} \mathrm{BF}_{4}$. The additive electrolyte included sulfolane (SL) and the additive materials fluoro-ethylene carbonate (FEC) and vinyl ethylene carbonate (VEC). Moisture was removed from the electrolyte using molecular sieves, which were dried at $120^{\circ} \mathrm{C}$ for $24 \mathrm{~h}$.

\subsection{Characterization}

All electrolyte preparations and cell assemblies were conducted in an Ar-filled glove box (koreakiyon, Korea). When assembling the three-electrode systems, the base electrolyte was EC:DMC (vol. ratio $=1: 1)$ with $1 \mathrm{M} \mathrm{SP}_{2} \mathrm{BF}_{4}$. Electrochemical impedance spectra (EIS, ZIVE sp2, WonATech) were measured from $100 \mathrm{kHz}$ to $30 \mathrm{mHz}$ with an amplitude of $10 \mathrm{mV}$ at the open circuit voltage. The surface morphologies of electrodes after cycling were observed using high resolution-transmission electron micros- copy (HR-TEM, JEM 2000EX, JEOL).

\section{Results and Discussion}

Fig. 1 shows the Nyquist plots of EDLCs with $1 \mathrm{M}$ $\mathrm{SP}_{2} \mathrm{BF}_{4}$-based (a) EC:DMC, (b) EC:DMC + SL $20 \%$, (c) EC:DMC + SL $20 \%+$ FEC $1 \%$, and (d) $\mathrm{EC}: \mathrm{DMC}+\mathrm{SL} 20 \%+$ VEC $1 \%$ electrolytes at a frequencies between $100 \mathrm{kHz}$ and $30 \mathrm{mHz}$ at open circuit voltage. Nyquist plots of impedance spectra are a powerful technique that gives information on the internal resistance between the electrode and the electrolyte [11]. Generally, Nyquist plots of supercapacitors exhibit a high-frequency region and a lowfrequency region. In the high-frequency region shows the resistance of the electrolyte solution $\left(R_{s}\right)$, whlie the low-frequency region shows the charge transfer resistance $\left(\mathrm{R}_{\mathrm{ct}}\right)$ and ion diffusion (W) inclined at a constant angle to the real axis [12]. The $\mathrm{R}_{\mathrm{s}}$ values of EC:DMC, EC:DMC + SL $20 \%$, $\mathrm{EC}: \mathrm{DMC}+\mathrm{SL} 20 \%+\mathrm{FEC} 1 \%$, and EC:DMC + SL $20 \%+$ VEC $1 \%$ at open circuit voltage were observed to be $2.05,2.02,1.79$, and $1.47 \Omega$ respectively. The $R_{s}$ depends on the conductivity of the electrolyte, and the conductivity changes with the concentration of the electrolyte [11]. The $\mathrm{R}_{\mathrm{s}}$ values of $\mathrm{EC}: \mathrm{DMC}+\mathrm{SL} 20 \%$, EC:DMC + SL $20 \%+\mathrm{FEC}$ $1 \%$, and $\mathrm{EC}: \mathrm{DMC}+\mathrm{SL} 20 \%+\mathrm{VEC} 1 \%$ were lower than that of the EC:DMC electrolyte because

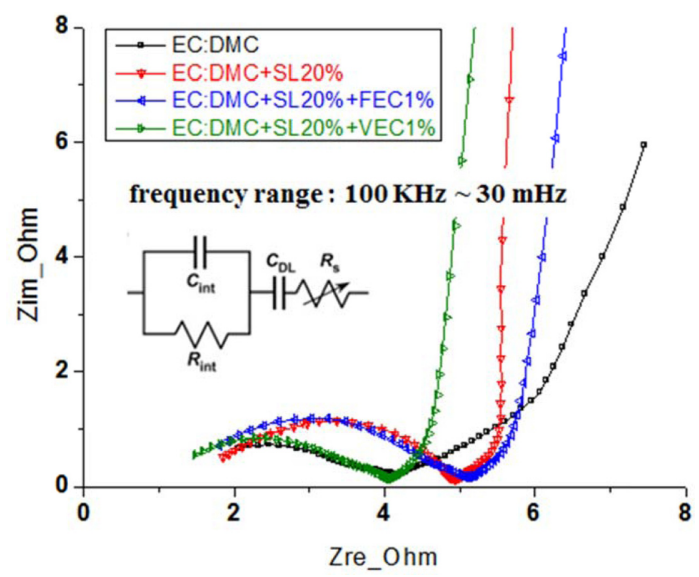

Fig. 1. Nyquist plots of EDLCs using $1 \mathrm{M} \mathrm{SP}_{2} \mathrm{BF}_{4}$-based EC:DMC, EC:DMC + SL $20 \%$, EC:DMC + SL $20 \%+$ FEC $1 \%$ and EC:DMC + SL $20 \%+$ VEC $1 \%$ electrolytes at frequencies range between $100 \mathrm{kHz}$ and $30 \mathrm{mHz}$ at the open circuit voltage. 
SL has a higher dielectric constant $\left(42.5 \mathrm{C}^{2} / \mathrm{m}^{2}\right)$ than DMC $\left(3.1 \mathrm{C}^{2} / \mathrm{m}^{2}\right)$. The $\mathrm{R}_{\mathrm{ct}}$ values of EC:DMC, $\mathrm{EC}: \mathrm{DMC}+\mathrm{SL} 20 \%$, EC:DMC + SL $20 \%+\mathrm{FEC}$ $1 \%$, and EC:DMC + SL $20 \%+$ VEC $1 \%$ were observed to be $2.06,2.99,3.22$, and $2.64 \Omega$ respectively. The $\mathrm{R}_{\mathrm{ct}}$ depends on the resistance at the interface between the electrode and electrolyte. [12] When additive materials were included, the $\mathrm{R}_{\mathrm{ct}}$ increased compared to that of the EC:DMC electrolyte. This suggests that initial charge and discharge capacitance were reduced compared to EC:DMC, but cycle retention will be expected the reduced resistance of the solution.

Fig. 2 shows cycle performance of the EDLCs with $1 \mathrm{M} \mathrm{SP}_{2} \mathrm{BF}_{4}$-based (a) EC:DMC, (b) EC:DMC + SL $20 \%$, (c) EC:DMC + SL $20 \%+$ FEC $1 \%$, and (d) $\mathrm{EC}: \mathrm{DMC}+\mathrm{SL} 20 \%+$ VEC $1 \%$ electrolytes at 0 $3.5 \mathrm{~V}$ at a current density of $1.52 \mathrm{~A} / \mathrm{g}$. The initial discharge capacitances of EC:DMC SL $20 \%$, EC:DMC + SL $20 \%+$ FEC $1 \%$, and EC:DMC + SL $20 \%+$ VEC $1 \%$ were decreased due to the presence of the additive materials. However, cycle retentions were enhanced from $64 \%$ to $88 \%, 96 \%$, and $94 \%$, respectively, after 500 cycles. As shown in Fig. 1, increasing the $\mathrm{R}_{\mathrm{ct}}$ reduced the initial discharge, but improved cycle retention was expected due to the reduced $\mathrm{R}_{\mathrm{s}}$. Additionally, reductive decomposition of FEC and VEC before EC:DMC was expected to due to their lower LUMO levels $(-0.12 \mathrm{eV}$ and $-0.5 \mathrm{eV}$, respectively) and higher HOMO levels (-7.79 eV and $7.75 \mathrm{eV}$, respectively) than EC (LUMO: $0.86 \mathrm{eV}$ and HOMO: $-8.19 \mathrm{eV}$ ). This allows them to form the stable

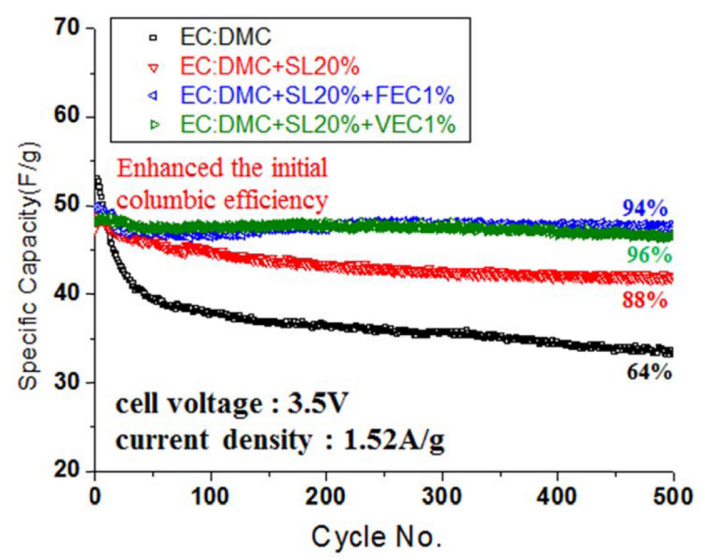

Fig. 2. Cycle performances of EDLCs with $1 \mathrm{M} \mathrm{SP}_{2} \mathrm{BF}_{4}$ based electrolytes at $0-3.5 \mathrm{~V}$ at a current density of $1.52 \mathrm{~A} / \mathrm{g}$.
SEI layers by reacting at higher potentials than EC.

Fig. 3 shows the reductive degradation mechanisms of FEC and VEC at high voltages $(>3.0 \mathrm{~V})$. According to the work of Kim et al., FEC can reductively decompose into vinylene carbonate (VC) and $\mathrm{HF}$ [9]. $\mathrm{VC}$ is easily reduced and tends to polymerize due to the presence the double bond between the carbon atoms. Such polymerized SEI films prevent further decomposition of the electrolyte. VEC is also capable of reductive decomposition and formation of stable SEI films [1315]. Reductive degradation is expected to prevent the degradation of EC:DMC due to the lower LUMO levels and higher HOMO levels of the materilas than EC.

Fig. 4 shows TEM images of the electrode surfaces of EDLCs with $1 \mathrm{M} \mathrm{SP}_{2} \mathrm{BF}_{4}$-based (a) EC:DMC, (b) $\mathrm{EC}: \mathrm{DMC}+\mathrm{SL} 20 \%$ and (c) EC:DMC + SL $20 \%+$ VEC $1 \%$ after 100 cycles. In TEM images, it can be seen that an amorphous layer had been generated on the surface of the active carbon electrode after 100 cycles. When additive materials were included along with EC:DMC the base electrolyte, the thickness of the SEI layer increased from $22 \mathrm{~nm}$ to $83 \mathrm{~nm}$. It is suggested that the formation of the SEI layer during cycling improved performance at high voltages. The stable SEI layer protected the electrode surface

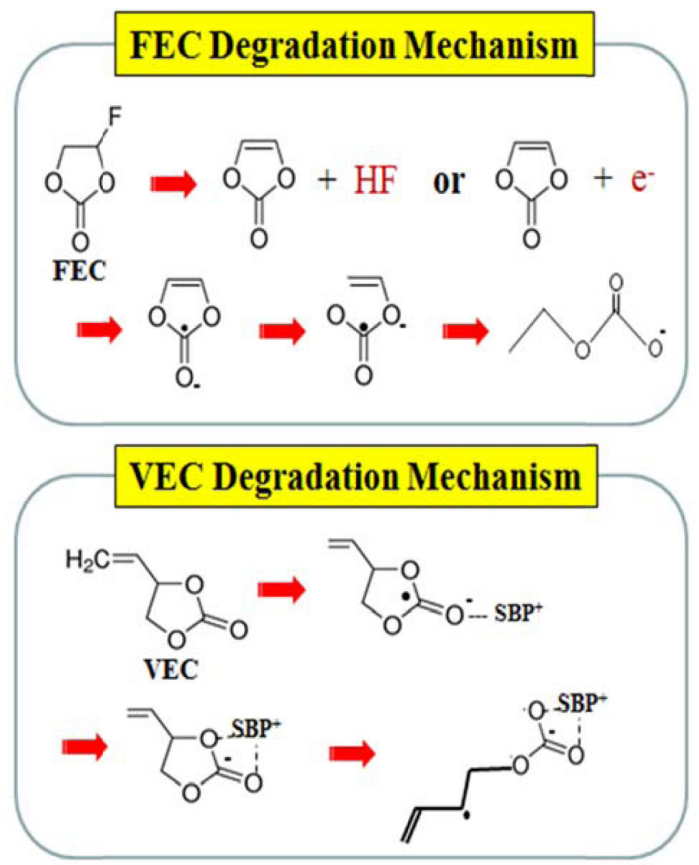

Fig. 3. Degradation mechanisms of FEC and VEC at high voltages $(>3.0 \mathrm{~V})$. 
(a) EC:DMC

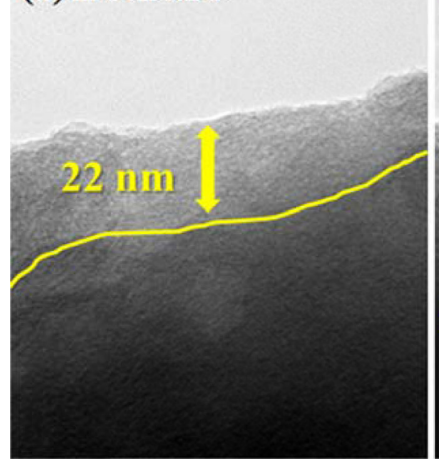

(b) EC:DMC + SL 20\%

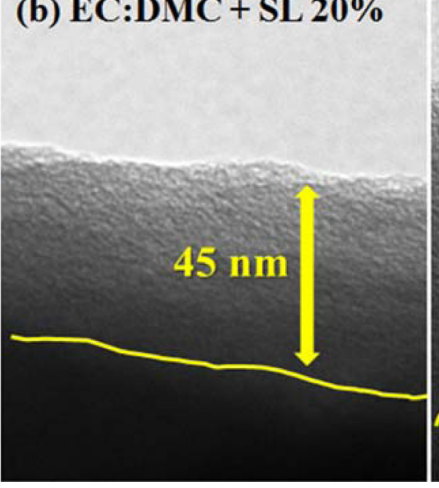

(c) EC:DMC + SL20\%

+ VEC $1 \%$

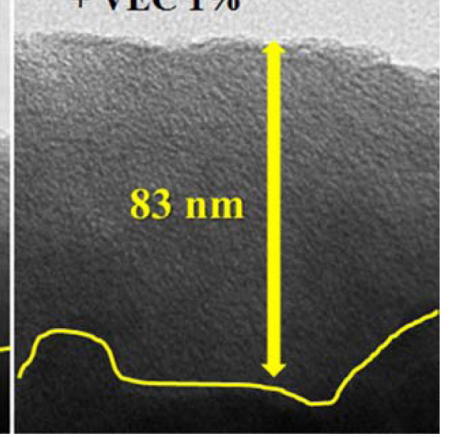

Fig. 4. TEM images of the electrode surfaces of EDLCs with $1 \mathrm{M} \mathrm{SP}_{2} \mathrm{BF}_{4}$-based (a) EC:DMC, (b) EC:DMC + SL $20 \%$, and (c) EC:DMC + SL $20 \%$ + VEC $1 \%$ electrolytes after 100 cycles.

during cycling performance. Additionally, it prevented degradation of the EC:DMC base electrolyte at high voltages, which could allow development of electrochemical capacitors with high energy densities.

\section{Conclusion}

We studied the possibility of increasing the working voltage of EDLCs in order to enhance the energy density of electrochemical capacitors. To prevent the decomposition of the electrolyte, stable SEI layers were formed by reductive degradation of additive materials such as FEC and VEC, which prevented degradation of the base electrolyte. As a result, $\mathrm{R}_{\mathrm{s}}$ value of the EC:DMC + SL $20 \%+$ VEC $1 \%$ electrolyte was $1.47 \Omega$ and $\mathrm{R}_{\mathrm{ct}}$ value was $2.64 \Omega$ at the open circuit voltage. Additionally, the cycle retention of the EC:DMC + SL $20 \%+$ VEC $1 \%$ electrolyte was observed $94 \%$ after 500 cycles at $3.5 \mathrm{~V}$ at a current density of $1.52 \mathrm{~A} / \mathrm{g}$. TEM images show that an amorphous material formed on electrode surface. However, further work will be needed to determine the precise formation mechanism of the SEI layer and its constituents.

\section{Acknowledgement}

This work was supported by the intramural research grant of Chungbuk National University in 2013 and the Korea Institute of Energy Technology Evaluation and Planning (KETEP) and the Ministry of Trade, Industry \& Energy (MOTIE) of the Republic of Korea (No. 20142020104160).

\section{References}

[1] C. W. Liew, S. Ramesh and A. K. Arof, Mater. Design, 2016, 92, 829-835.

[2] G. Srikeshz and A. Samson Nesaraj, J. Electrochem. Sci. Technol., 2015, 6, 16-25.

[3] Z. K. Ghouri, M. S. Akhtar, A. Zahoor, N. A. M. Barakat, W. Han, M. Park, B. Pant, P. S. Saud, C. H. Lee and H. Y. Kim, J. Alloys Compd., 2015, 642, 210215.

[4] J. J. Yang, Y. R. Kim, M. G. Jeong, Y. J. Yuk, H. J. Kim and S. G. Park, J. Electrochem. Sci. Technol., 2015, 6, 59-64.

[5] G. A. Tiruye, D. M. Torrero, J. Palma, M. Anderson and R. Marcilla, J. Power Sources, 2015, 279, 472-480.

[6] Q. Abbas, P. Babuchowska, E. Frackowiak and F. Bleguin, J. Power Sources, 2016, in press.

[7] S. Pohlmann, T. Olyschlager, P. Goodrich, J. Alvarez Vicente, J. Jacquemin and A. Balducci, J. Power Sources, 2015, 273, 931-936.

[8] L. Xing, J. Vatamanu, O. Borodin, G. D. Smith and D. Bedrov, J. Phys. Chem. C, 2012, 116, 23871-23881.

[9] M. Kim, I.-J. Kim, S. Yang and S. Kim, Bull. Korean Chem. Soc., 2014, 35, 466-470.

[10] R. Wang, X. Li, Z. Wang, H. Guo, J. Wang and T. Hou, J. Alloys Compd., 2015, 632, 435-444.

[11] S. A. Delp, O. Borodin, M. Olguin, C. G. Eisner, J. L. Allen and T. R. Jow, Electrochim. Acta, 2016, 209, 498510 .

[12] W. Liu, X. Yan, J. Lang and Q. Xue, J. Mater. Chem., 2012, 22, 8853-8861.

[13] S. D. Xu, Q. C. Zhuang, J. Wang, Y. Q. Xu and Y. B. Zhu, Int. J. Electrochem. Sci., 2013, 8, 8058-8076.

[14] J. Li, W. Yao, Y. S. Meng and Y. Yang, J. Phys. Chem. $C$, 2008, 112, 12550-12556.

[15] Y. Hu, W. Kong, H. Li, X. Huang and L. Chen, Electrochem. Commun., 2004, 6, 126-131. 